ARTICLE

DOI: $10.1038 / s 41467-018-05932-9$

\title{
Measurement of complex optical susceptibility for individual carbon nanotubes by elliptically polarized light excitation
}

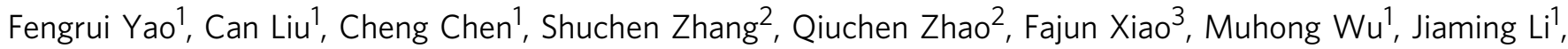
Peng Gao (1) ${ }^{4}$, Jianlin Zhao ${ }^{3}$, Xuedong Bai (1) ${ }^{5}$, Shigeo Maruyama (i] ${ }^{6,7}$, Dapeng Yu (D) ${ }^{8}$, Enge Wang ${ }^{4}$, Zhipei Sun (10) ${ }^{9,10}$, Jin Zhang (10) ${ }^{2}$, Feng Wang ${ }^{11} \&$ Kaihui Liu (i) ${ }^{1}$

The complex optical susceptibility is the most fundamental parameter characterizing lightmatter interactions and determining optical applications in any material. In one-dimensional (1D) materials, all conventional techniques to measure the complex susceptibility become invalid. Here we report a methodology to measure the complex optical susceptibility of individual 1D materials by an elliptical-polarization-based optical homodyne detection. This method is based on the accurate manipulation of interference between incident left- (right-) handed elliptically polarized light and the scattering light, which results in the opposite (same) contribution of the real and imaginary susceptibility in two sets of spectra. We successfully demonstrate its application in determining complex susceptibility of individual chirality-defined carbon nanotubes in a broad optical spectral range (1.6-2.7 eV) and under different environments (suspended and in device). This full characterization of the complex optical responses should accelerate applications of various 1D nanomaterials in future photonic, optoelectronic, photovoltaic, and bio-imaging devices.

\footnotetext{
${ }^{1}$ State Key Laboratory for Mesoscopic Physics, Collaborative Innovation Centre of Quantum Matter, School of Physics, Peking University, Beijing 100871, China. ${ }^{2}$ Center for Nanochemistry, College of Chemistry and Molecular Engineering, Peking University, Beijing 100871, China. ${ }^{3}$ School of Science, Northwestern Polytechnical University, Xi'an 710129, China. ${ }^{4}$ International Center for Quantum Materials and Electron Microscopy Laboratory, Peking University, Beijing 100871, China. ${ }^{5}$ Institute of Physics, Chinese Academy of Sciences, Beijing 100875, China. ${ }^{6}$ Department of Mechanical Engineering, The University of Tokyo, Tokyo 113-8656, Japan. ${ }^{7}$ Energy NanoEngineering Lab, National Institute of Advanced Industrial Science and Technology, Tsukuba 3058564, Japan. ${ }^{8}$ Department of Physics, Southern University of Science and Technology, Shenzhen 518055, China. ${ }^{9}$ Department of Electronics and Nanoengineering, Aalto University, Espoo 02150, Finland. ${ }^{10}$ QTF Centre of Excellence, Department of Applied Physics, Aalto University, Espoo 02150 , Finland. ${ }^{11}$ Department of Physics, University of California at Berkeley, Berkeley, CA 94720, USA. These authors contributed equally: Fengrui Yao, Can Liu, Cheng Chen. Correspondence and requests for materials should be addressed to K.L. (email: khliu@pku.edu.cn)
} 
O ne-dimensional (1D) materials are at the center of significant research effort of nano science and technology. For example, carbon nanotubes, a 1D material from rolled-up graphene, have shown fascinating optical properties, e.g., quantized optical transitions ${ }^{1-3}$, strong many-body interactions $^{4-9}$ and efficient photon-electron generation ${ }^{10}$, thus enabling diverse applications ranging from photonics ${ }^{11-14}$, optoelectronics $^{15-17}$, and photovoltaics ${ }^{18}$ to bio-imaging ${ }^{19}$. To fully utilize 1D materials for various application, we need to know their optical response parameters that quantitatively describe lightmatter interactions, among which the complex optical susceptibility $(\tilde{\chi})$ is the most fundamental one ${ }^{20,21}$ (describing the response of the dipole moment $\mathbf{P}$ of crystalline materials to external optical field $\left.\mathbf{E}, \mathbf{P}=\tilde{\chi} \varepsilon_{0} \mathbf{E}, \tilde{\chi}=\tilde{\epsilon}-1\right)$. Unlike mature technology for measuring the complex optical susceptibility by solving Fresnel equations in two-dimensional (2D) and threedimensional (3D) materials ${ }^{22}$, there is no available technique to effectively measure both the real and imaginary parts of $\tilde{\chi}$ in individual $1 \mathrm{D}$ materials. The main difficulty lies in the vanishing of concept for coherent refraction or reflection and thus conventional methodologies become invalid ${ }^{23}$. One way to circumvent this difficulty is to measure the absorption of $1 \mathrm{D}$ materials (e.g., carbon nanotubes ${ }^{24-26}$ ), which is proportional to the imaginary part susceptibility $\left(\chi_{2}\right)$. By employing the Kramers-Kronig $(\mathrm{K}-\mathrm{K})$ relation, one can in principle calculate the real part susceptibility $\chi_{1}$. However, to get the accurate value, the application of $\mathrm{K}-\mathrm{K}$ relation requires experimental data of $\chi_{2}$ over an extremely broad energy region from $0 \mathrm{eV}$ to infinity, which is never available. Until now, there is no method to directly measure the complex optical susceptibility of individual 1D materials.

Here we develop a methodology to measure the complex optical susceptibility for individual carbon nanotubes by an elliptical-polarization-based optical homodyne detection. By accurately controlling the interference between incident lefthanded and right-handed elliptical polarization beam and nanotube-scattering light, we obtain two sets of optical spectra containing both $\chi_{1}$ and $\chi_{2}$ information with different pre-coefficients, which allow us to determine the quantitative value of $\tilde{\chi}$. In addition, our technique also enhances the optical signal level by about two orders of magnitude, making the extremely weak individual nanotube signal readily detectable in a broad optical spectral range $(1.6-2.7 \mathrm{eV})$ and in different environments (suspended and in device). Our results can open up exciting opportunities in characterizing a variety of $1 \mathrm{D}$ nanomaterials, including graphene nanoribbon, nanowires, and other nano-biomaterials, thus facilitating their accurate material design and applications in future photonic, optoelectronic, photovoltaic, and bio-imaging devices.

\section{Results}

Scheme of complex optical susceptibility measurement in a transmission geometry. For individual nanotubes with diameter $(\sim 1 \mathrm{~nm})$ much less than light wavelength $(\sim 500 \mathrm{~nm})$, their optical signal in reflection/transmission geometry can be viewed as the interference between optical reference and nanotube-scattering fields ${ }^{27,28}$. To obtain both $\chi_{1}$ and $\chi_{2}$ of a nanotube, in principle we need two optical reference components with a $\pi / 2$ phase difference. Actually, circularly/elliptically polarized light naturally provides such a $\pi / 2$ phase difference, which has been proved to be an effective excitation for optical tomography ${ }^{29}$, spin/quantum computing and information ${ }^{30}$, valleytronics ${ }^{31}$, and highharmonic generation ${ }^{32}$. In our work, we choose elliptical polarization light with a relatively large ellipticity as excitation. Its advantage over circularly polarized light lies in that the reference beam can be greatly reduced by two vertically placed polarizers, which will greatly enhance the final optical contrast as demonstrated in the previous single-tube Rayleigh scattering, absorption and reflection measurement using linear polarization excitation light $^{26,33,34}$.

We first apply this technique to individual suspended singlewalled carbon nanotubes in a transmission geometry. Experimental scheme of our elliptical-polarization-based optical homodyne detection method is shown in Fig. 1a. A supercontinuum laser was used as the light source to provide a broadband excitation $(450-900 \mathrm{~nm})$, a pair of confocal polarization-maintaining objectives served to focus the supercontinuum light on individual nanotubes and collected the transmitting and nanotube-scattering lights, two polarizers and a quarter-wave plate were used to generate elliptically polarized light and control the polarization of transmitted light. Here we should note that the very careful selection of objectives and wave plate to maintain the polarization purity is crucial to realize the final signal detection (See optical component details in Methods). Layouts of the polarization control are shown in Fig. 1b, c. The two polarizers were set strictly perpendicular to each other, and the suspended nanotube was positioned at an angle of $\pi / 4$ with respect to two polarizers. In the two different configurations, fast axis of the quarter-wave plate was kept at a small angle $(\theta)$ with polarizer 1 (Fig. 1b) or polarizer 2 (Fig. 1c) to generate left-handed $\left(\boldsymbol{E}_{\mathbf{L}}\right)$ or right-handed $\left(\boldsymbol{E}_{\mathbf{R}}\right)$ ellipticalpolarization light as the excitation for the nanotube. Due to the very strong $1 \mathrm{D}$ depolarization effect, the nanotube-scattering field is mainly polarized along the nanotube axial direction ${ }^{35,36}$. Therefore, the nanotube forward-scattering field can be written as $E_{\mathrm{NT}}^{\mathrm{L}}=\beta \tilde{\chi} E_{\mathrm{L}}$ and $E_{\mathrm{NT}}^{\mathrm{R}}=\beta \tilde{\chi} E_{\mathrm{R}}$, respectively, where $\beta$ represents a scattering coefficient. Before polarizer 2 , the forwardscattering light of the nanotube interferes with the transmitted optical reference beam (Fig. 1d, e), which yields the optical contrast signal as

$$
\frac{\Delta T_{\mathrm{i}}}{T}=\frac{\left|E_{\mathrm{i}}+E_{\mathrm{NT}}^{\mathrm{i}}\right|^{2}-\left|E_{\mathrm{i}}\right|^{2}}{\left|E_{\mathrm{i}}\right|^{2}} \approx \frac{2 \operatorname{Re}\left(E_{\mathrm{i}} E_{\mathrm{NT}}^{\mathrm{i}}\right)}{\left|E_{\mathrm{i}}\right|^{2}},
$$

where $T_{\mathrm{i}}$ is the transmission signal intensity, $\Delta T_{\mathrm{i}}$ is the change of transmission signal intensity resulted from the presence of a nanotube, and i stands for $\mathrm{L}$ or $\mathrm{R}$ (The $\left|E_{\mathrm{NT}}^{\mathrm{i}}\right|^{2}$ term has been neglected because it is orders of magnitude smaller than the cross term). After polarizer 2, the reference light field is decreased dramatically due to small eccentricity of elliptically polarized light $\left(E_{\mathrm{o}}^{\mathrm{i}}=\left(E_{\mathrm{i}} \sin 2 \theta\right) / \sqrt{2}\right)$, while the nanotube's scattering field is only decreased by a small proportion $\left(E_{\mathrm{s}}^{\mathrm{i}}=E_{\mathrm{NT}}^{\mathrm{i}} / \sqrt{2}\right)$. Therefore, the optical contrast is greatly enhanced, typically by 20 times in our transmission geometry. More importantly, the variation of the modulated signals depends on the real part of the cross term $E_{\mathrm{o}}^{\mathrm{i}}\left(E_{\mathrm{s}}^{\mathrm{i}}\right)^{*}$, and this term varies with the elliptical chirality of incident light (Fig 1f, g). In detail, detected optical contrast signals caused by left- or righthanded incident light can be written as:

$$
\frac{\Delta T_{\mathrm{L}}}{T}=\alpha\left(\chi_{2}-\chi_{1}\right), \frac{\Delta T_{\mathrm{R}}}{T}=\alpha\left(\chi_{2}+\chi_{1}\right), \alpha=\frac{\beta}{\sin 2 \theta} .
$$

Since $\chi_{1}$ and $\chi_{2}$, respectively, have the opposite and same contribution in the optical signal with left- and right-handed elliptically polarized excitation, the solution of both $\chi_{1}$ and $\chi_{2}$ can be obtained unambiguously (See more details in Supplementary Note 1).

Complex optical susceptibility measurement of individual suspended nanotube. Scanning electron microscopic image of a 


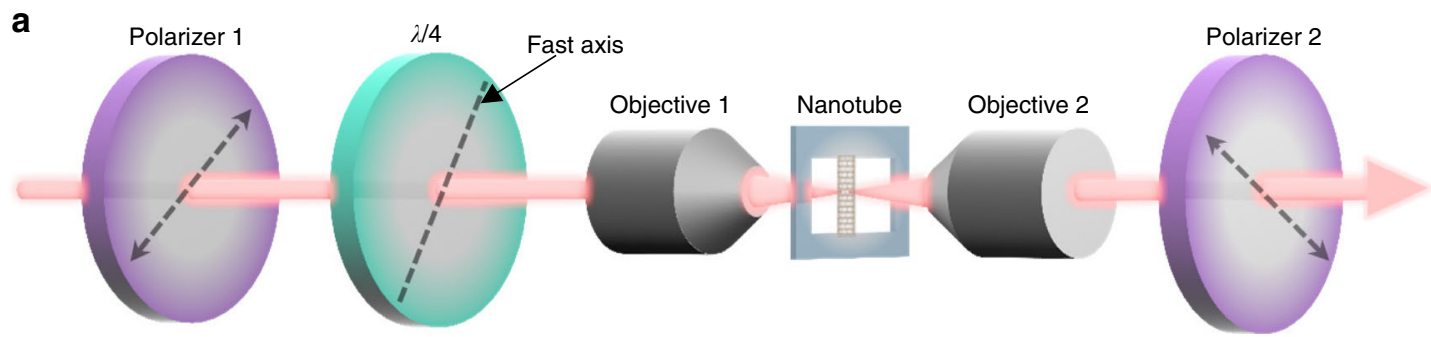

b

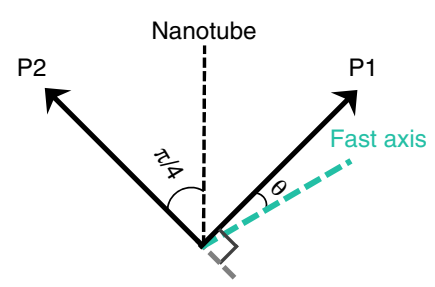

C

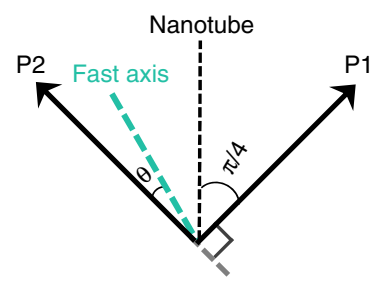

d

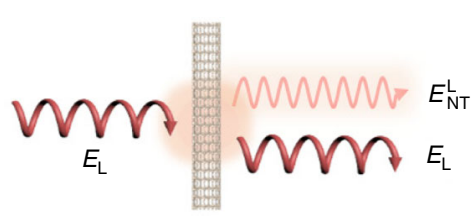

e

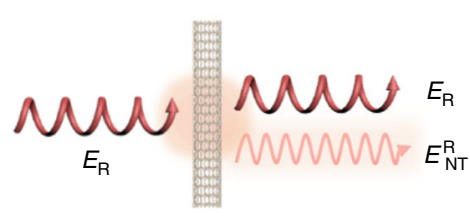

f

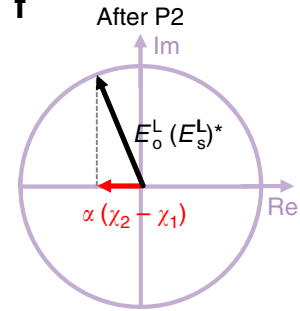

g

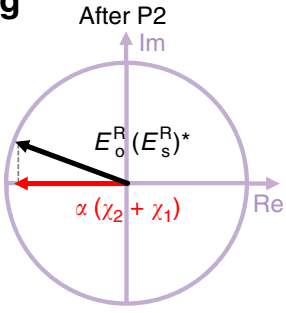

Fig. 1 Scheme of complex optical susceptibility measurement in a transmission geometry. a Scheme of experiment setup. Two polarizers were strictly perpendicular to each other. A quarter-wave plate was used to generate the elliptical chirality (left- or right-handed). A vertically placed carbon nanotube was put at the focus of the two confocal objectives. $\mathbf{b}$, $\mathbf{c}$ Layouts of the polarization control and the nanotube. The nanotube was laid at the bisector of the two polarizers' axis. The fast axis of the wave plate was kept at a small angle $(\theta)$ to the polarizer axis. Polarizer 2 and polarizer 1 were abbreviated to $P 2$ and P1. d, e Interference scheme of input left- $\left(E_{\mathrm{L}}\right)$ and right-handed $\left(E_{\mathrm{R}}\right)$ elliptically polarized light and nanotube forward-scattering field $\left(E_{\mathrm{NT}}^{\mathrm{L}}\right.$ or $\left.E_{\mathrm{NT}}^{\mathrm{R}}\right)$.

$\mathbf{f}, \mathbf{g}$ Illustrations of complex phase diagrams of the detected optical contrast signal with left/right elliptically polarized light excitation after polarizer 2 , in which $\chi_{1}$ contributes oppositely under two layouts
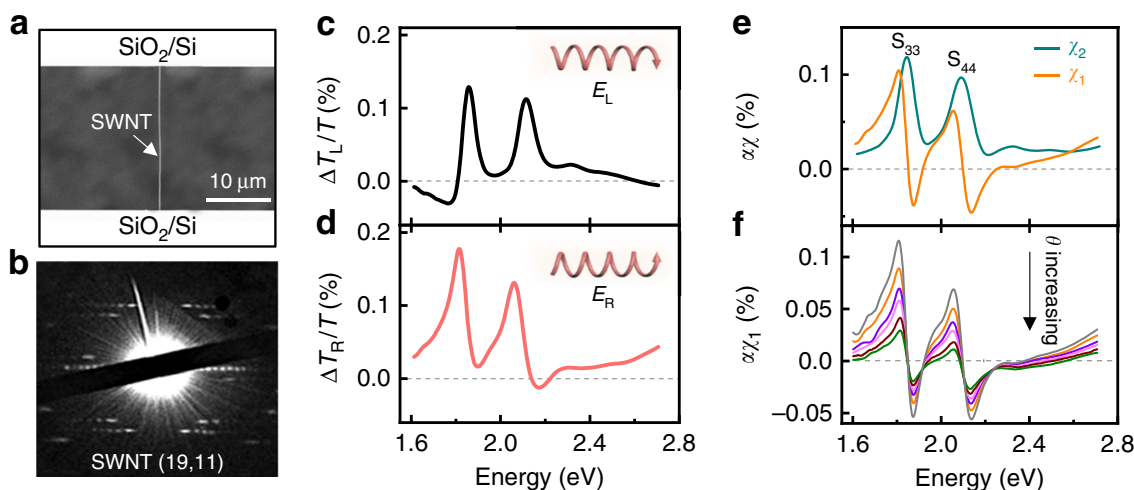

Fig. 2 Complex optical susceptibility measurement of individual suspended nanotube. a Scanning electron micrograph (SEM) image of a suspended carbon nanotube across an open slit etched on $\mathrm{SiO}_{2} / \mathrm{Si}$ substrate. $\mathbf{b}$ The electron diffraction pattern reveals the chiral index of nanotube as (19, 11), a semiconducting tube with a diameter of $2.06 \mathrm{~nm}$. c, $\mathbf{d}$ Optical contrast spectra with left- $\left(E_{\mathrm{L}}\right)(\mathbf{c})$ and right-handed $\left(E_{\mathrm{R}}\right)$ (d) elliptically polarized excitation. The angle $\theta$ between the wave plate and polarizer 1 or polarizer 2 is set as $2^{\circ}$. e Real ( $\alpha \chi_{1}$, orange line) and imaginary ( $\alpha \chi_{2}$, green line) susceptibility of the nanotube under $\theta=2^{\circ}$. The two peaks are corresponded to $S_{33}$ and $S_{44}$ optical transitions, respectively. $\mathbf{f}$ Dependence of detected real susceptibility value $\left(\alpha \chi_{1}\right)$ on $\theta$. With $\theta$ increasing from $1.4^{\circ}$ to $4^{\circ}$, the signal decreases linearly with $1 / \sin 2 \theta$ (Supplementary Fig. 1). $\alpha$ is a detection coefficient

nanotube suspended across an open slit in $\mathrm{SiO}_{2} / \mathrm{Si}$ substrate is shown in Fig. 2a. The chiral index of this nanotube was identified as $(19,11)$ from its electron diffraction pattern (Fig. 2b) and its Rayleigh scattering or absorption spectrum ${ }^{37}$. Figure $2 \mathrm{c}, \mathrm{d}$ shows optical contrast signals in photon energy range of $1.60-2.70 \mathrm{eV}$ under left- (Fig. 2c) and right-handed (Fig. 2d) elliptically polarized light excitation $\left(\theta=2^{\circ}\right)$. This set of spectra show two characteristic features, which are significantly different from absorption spectra ${ }^{26}$ : (1) the peaks are not Lorentzian like, with slow bumps and pits; (2) optical contrast signal goes below zero at 
a

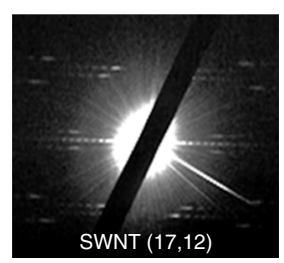

d

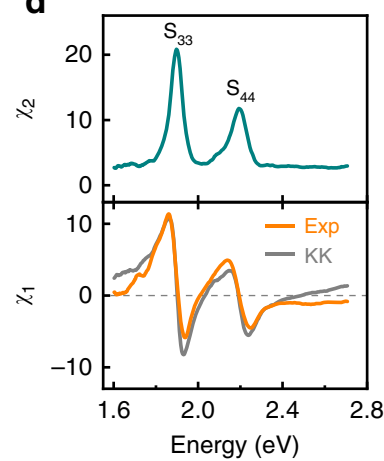

b

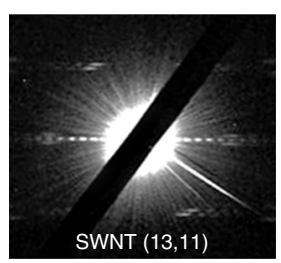

e

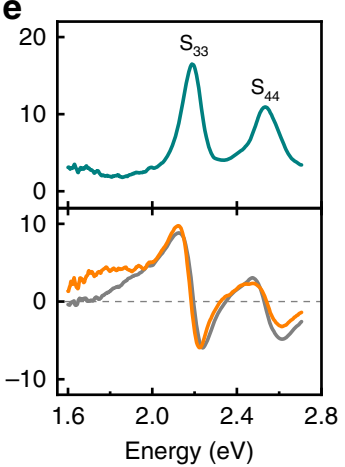

c

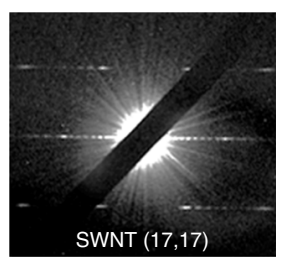

f

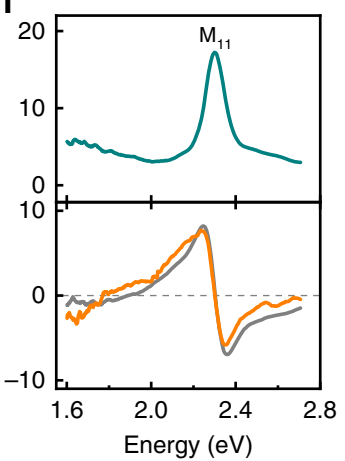

Fig. 3 Systematical complex optical susceptibility measurement of individual nanotubes. a-c Electron diffraction patterns of three single-walled carbon nanotubes with different chiral indices. The $(17,12)$ semiconducting nanotube $(\mathbf{a})$ has a diameter of $1.98 \mathrm{~nm}$; the $(13,11)$ semiconducting nanotube $(\mathbf{b})$ has a diameter of $1.63 \mathrm{~nm}$; the $(17,17)$ metallic nanotube $(\mathbf{c})$ has a diameter of $2.31 \mathrm{~nm}$. $\mathbf{d}$-f, Measured imaginary $\left(\chi_{2}\right.$, green) and real $\left(\chi_{1}\right.$, orange) susceptibility of nanotubes in a-c under $\theta=2^{\circ}$. Optical transitions types are marked above each peak. The calculated real susceptibility ( $\chi_{1}^{\mathrm{KK}}$, gray) through Kramers-Kronig transformation of $\chi_{2}$ in a finite photon energy range (1.6-2.7 eV) were also shown. A good agreement between $\chi_{1}$ and $\chi_{1}^{\mathrm{KK}}$ is achieved around the resonance peak region, while obvious deviation can happen in non-resonant region. Optical transitions are marked above each peak

some energies. These features are originated from the opposite (same) contribution of $\chi_{1}\left(\chi_{2}\right)$ to the optical signal. According to Eq. 2, we extract the spectra of $\chi_{1}$ (orange line) and $\chi_{2}$ (green line) separately (Fig. 2e). The two optical resonances sit at 1.83 and $2.09 \mathrm{eV}$, corresponding to $\mathrm{S}_{33}$ and $\mathrm{S}_{44}$ optical transitions of the nanotube. The main peaks of $\chi_{2}$ can be decomposed into the dominant exciton contribution and a continuum contribution (band-to-band transitions) ${ }^{26}$. The weak peak located at $2.3 \mathrm{eV}$ above the main resonance is attributed to a phonon side band ${ }^{38-41}$. In addition to solving $\chi_{1}$ and $\chi_{2}$, the elliptical polarization excitation also enables the enhancement of the detected contrast signal as we accurately control the fast axis angle $\theta$. When $\theta$ increases from $1.4^{\circ}$ to $4^{\circ}$, the detected signal (taking $\alpha \chi_{1}$ as an example) decreases linearly with $1 / \sin 2 \theta$ (Fig. $2 \mathrm{f}$ and Supplementary Fig. 1), which is in accordance with the quantitative analysis of Eq. 2.

Systematic complex optical susceptibility measurement of individual nanotubes. By estimating the scattering coefficient $\beta$ in our experiment (See Supplementary Note 2), we can further give out the absolute value of $\tilde{\chi}$ for a nanotube. In total, 20 singlewalled carbon nanotubes are measured (Fig. 3 and Supplementary Fig. 2 and Fig. 3) and three representative complex susceptibilities of them are shown in Fig. 3. From the electron diffraction patterns and optical transitions (Fig. 3a-c), these three nanotubes are identified as semiconducting $(17,12)$ and $(13,11)$ and metallic $(17,17)$, respectively. Obviously, both $\chi_{2}$ and $\chi_{1}$ spectra (Fig. $3 \mathrm{~d}-\mathrm{f}$ ) are intrinsic characteristics of a nanotube. As the ultralong nanotubes on open slits are typically with diameters of 1.5-3.0 $\mathrm{nm}$, we can only access optical transitions higher than $\mathrm{S}_{22}$ in our laser spectral range of $1.6-2.7 \mathrm{eV}$. In principle, we can also measure $\mathrm{E}_{11}$ transition as long as one can grow the ultralong nanotubes with diameter less than $0.8 \mathrm{~nm}$ on the wide slit in the future.
According to Kramers-Kronig relation, the $\chi_{1}$ can be obtained from $\chi_{2}$ by

$$
\chi_{1}(E)=\frac{2}{\pi} \int_{0}^{\infty} \frac{E^{\prime} \chi_{2}\left(E^{\prime}\right)}{\left(E^{\prime}\right)^{2}-E^{2}} d E^{\prime}
$$

This integration requires the full energy data ranging from $0 \mathrm{eV}$ to infinity of $\chi_{2}$. Nevertheless, by utilizing the measured $\chi_{2}$ data in energy range of $1.6-2.7 \mathrm{eV}$ to perform $\mathrm{K}-\mathrm{K}$ transformation, we can deduce a predicted $\chi_{1}^{\mathrm{KK}}$ (Fig. $3 \mathrm{~d}-\mathrm{f}$, gray lines). We found a relative good agreement between $\chi_{1}$ and $\chi_{1}^{\mathrm{KK}}$ around the resonance peak region, while obvious deviations in nonresonant region were observed. The agreement in the resonant region actually proves the accuracy of our measurement of $\chi_{1}$, because the denominator weight factor around resonance region in Eq. 3 ( $E^{\prime}$ is close to $E$ ) is very large and the ignorance of other non-resonant regions should still yield pretty good prediction. The agreement also implies the conventional susceptibility model describing optical responses still works even for such 1D nanostructures with many molecule-like optical properties. While the deviation in the non-resonant region highlights the necessity of independent $\chi_{1}$ measurement other than predicted $\chi_{1}^{\mathrm{KK}}$ since one could never obtain $\chi_{2}$ in full energy region. The discrepancy can be understood from Eq. 3. When conducting K-K transformation in a limited range of $1.6-2.7 \mathrm{eV}$, the influence of $\chi_{2}$ spectra outside the range is not considered, resulting in an inaccurate calculated value of $\chi_{1}^{\mathrm{KK}}$. $\chi_{2}$ below $1.6 \mathrm{eV}$ gives a negative contribution to calculated $\chi_{1}^{\mathrm{KK}}$, while $\chi_{2}$ above $2.7 \mathrm{eV}$ gives a positive contribution. Therefore, the accurate distribution of those unconsidered optical transitions mainly determines the deviation between $\chi_{1}$ and $\chi_{1}^{\mathrm{KK}}$. In particular, the optical transitions are fingerprints of each nanotube with different chirality, 
a

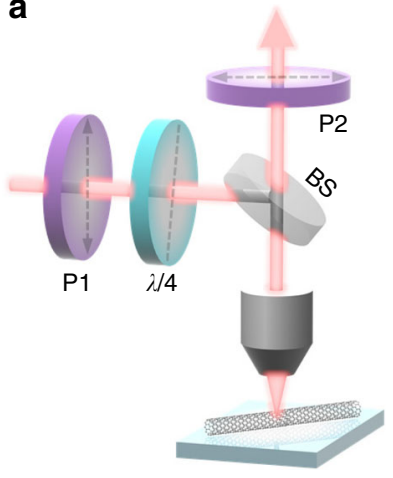

b

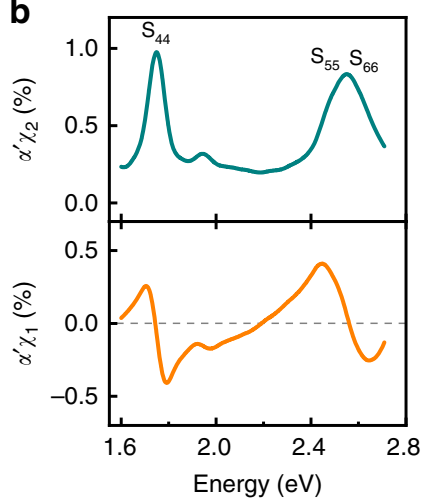

Fig. 4 On-chip complex optical susceptibility detection of individual nanotubes. a Scheme of the experiment setup in the reflection configuration. Beam splitter was abbreviated to BS. b Imaginary ( $\chi_{2}$, green) and real $\left(\chi_{1}\right.$, orange) susceptibilities of nanotube $(25,11)$ on fused quartz substrate. $\alpha^{\prime}$ is a detection coefficient. Optical transitions are marked above each peak. $\mathrm{S}_{55}$ and $\mathrm{S}_{66}$ transitions are very close with each other

therefore a simple $\mathrm{K}-\mathrm{K}$ calculation from $\chi_{2}$ of limited energy range in principle cannot produce accurate $\chi_{1}^{\mathrm{KK}}$ constantly.

On-chip complex optical susceptibility detection of individual nanotubes. As for most optical applications in devices, nanotubes will be on substrates. Furtherly, we developed our technique for nanotubes on substrates in a reflection geometry (Fig. 4a). Compared with the transmission geometry, a reflection pre-factor $(1+r)^{2} / r$ is added to final contrast signal $\left(\alpha^{\prime}=\alpha(1+r)^{2} / r\right)$, where $r$ is the reflection coefficient calculated from Fresnel equations and $1+r$ is the local field experienced by the nanotube (See more details in Supplementary Note 3). This will lead to even higher contrast signal enhancement to $\sim 100$ times, in contrast to the $\sim 20$ times enhancement for suspended nanotubes in the transmission geometry. In Fig. 4b, we show a representative measurement on semiconducting $(25,11)$ nanotubes with nice $\chi_{1}$ and $\chi_{2}$ spectra. Optical transition peaks identified from the extracted imaginary data were used to determine the nanotube chirality based on the atlas developed before ${ }^{37}$. Same as transmission spectrum, deduced $\chi_{1}^{\mathrm{KK}}$ agrees with the experimental value around the resonance peak region (See Supplementary Fig. 4).

\section{Discussion}

An advantage of carbon nanotubes for optical applications is the well-defined atomic structure and associated optical transitions that can be accurately described by both theory and experimental data bases ${ }^{37}$. From our $\tilde{\chi}$ measurement, we can clearly see that every nanotube has different $\chi_{1}$ and $\chi_{2}$ value at different energy regions, which is a great advantage in the nanophononics, including but not limited to metasurfaces. With the spatial control of the $\tilde{\chi}$-determined nanotubes, one can readily tailor the wave fronts of light beams with arbitrary phase, polarization and amplitude distributions, which holds a great promise to implement the subwavelength optical components ${ }^{42}$, optical computation $^{43}$, and information processing ${ }^{44}$, while enjoying the merits of the low loss and reduced dimension because the $1 \mathrm{D}$ nature of nanotubes. Further, nanotubes can have different walls, such as single wall and double walls with more optical transitions (See Supplementary Fig. 5), and the richness and flexibility in the optical engineering for meta-materials applications are therefore very promising ${ }^{45}$.
In summary, the direct measurement of the basic complex optical susceptibility of $1 \mathrm{D}$ materials is obviously fundamental for their accurate design and applications in the future photonic, optoelectronic, photovoltaic and bio-imaging devices, provides a new detectable parameter to monitor the external regulations such as charge doping, strain, molecular adsorption, and dielectric environment, and will further evoke theoretical understanding of $1 \mathrm{D}$ materials by complex susceptibility. The signal detection limit of our technique is estimated to be $\sim 10^{-6}$ (See Supplementary Note 4). This high sensitivity can ensure the detection of nanotubes with the smallest diameter down to $0.3 \mathrm{~nm}$. Thus, for general 1D materials with defined structure, such as long graphene nanoribbons and semiconductor nanowires, our technique is expected to work as well.

\section{Methods}

Nanotube preparation. In this study, nanotubes were grown by chemical vapor deposition method. We used ethanol through argon bubble as carbon precursor and a thin ion film $(0.2 \mathrm{~nm})$ as catalyst for the synthesis at $950{ }^{\circ} \mathrm{C}$ for $30 \mathrm{~min}$. Suspended SWNTs were grown across open slit structures on $\mathrm{SiO}_{2} / \mathrm{Si}$ substrate.

TEM (Transmission Electron Microscope) characterization. Electron diffraction patterns were obtained in TEMs (JEOL 2010F and JEM ARM 300CF) operated at $80 \mathrm{kV}$. Both electron beam and laser beam can go through the open slit with individual carbon nanotubes, which enables directly investigation of the chiral indices and optical spectrum of the same nanotube.

Optical setup. For transmission configuration (Fig. 1a), optical signal was collected by a home-built confocal microscope system, where a supercontinuum laser (Fianium SC-400-4) is used as the light source, shooting light through polarizer 1 (Thorlabs, GTH10M) and a quarter-wave plate (Thorlabs, AQWP05M-600A). Then an objective (Mitutoyo M Plan $50 \mathrm{X}, \mathrm{NA}=0.42$ ) serves to focus the light to the sample and another objective (Mitutoyo M Plan $50 \mathrm{X}, \mathrm{NA}=0.42$ ) collects the transmitted light. An oblique objective (Mitutoyo M Plan $50 \mathrm{X}, \mathrm{NA}=0.42$ ) was used to collect nanotube's scattering signal to a CCD camera (PULNIX TM-7CN) for Rayleigh imaging. The transmission signal was modulated by polarizer 2 (Thorlabs, GTH10M). Two sets of spectra with the nanotube in and out of the beam focus were obtained to generate the contrast spectra by a spectrometer containing a grating (Thorlabs, GT50-03) and a linear CCD (Imaging Solution Group, LW ELIS-1024a-1394). The Gaussian spatial profile of the light source was experimentally mapped by gradually changing the distance between the carbon nanotube and the beam focus (Supplementary Fig. 6). In the reflection geometry (Fig. 4a), the main difference is the use of a beam splitter (customer-polished quartz glass) and only one objective (Nikon S Plan Fluor $40 \mathrm{X}, \mathrm{NA}=0.65$ ).

Data availability. The authors declare that all relevant data are included in the paper and its Supplementary Information files. Additional data are available from the corresponding author upon reasonable request.

Received: 1 April 2018 Accepted: 31 July 2018

Published online: 23 August 2018

\section{References}

1. Saito, R., Fujita, M., Dresselhaus, G. \& Dresselhaus, M. S. Electronic-structure of chiral graphene tubules. Appl. Phys. Lett. 60, 2204-2206 (1992).

2. Kataura, H. et al. Optical properties of single-wall carbon nanotubes. Synth. Met. 103, 2555-2558 (1999).

3. Bachilo, S. M. et al. Structure-assigned optical spectra of single-walled carbon nanotubes. Science 298, 2361-2366 (2002).

4. Ando, T. Excitons in carbon nanotubes. J. Phys. Soc. Jpn. 66, 1066-1073 (1997).

5. Maultzsch, J. et al. Exciton binding energies in carbon nanotubes from twophoton photoluminescence. Phys. Rev. B. 72, 241402 (2005).

6. Wang, F., Dukovic, G., Brus, L. E. \& Heinz, T. F. The optical resonances in carbon nanotubes arise from excitons. Science 308, 838-841 (2005).

7. Matsunaga, R., Matsuda, K. \& Kanemitsu, Y. Evidence for dark excitons in a single carbon nanotube due to the Aharonov-Bohm effect. Phys. Rev. Lett. 101, 147404 (2008).

8. Colombier, L. et al. Detection of a biexciton in semiconducting carbon nanotubes using nonlinear optical spectroscopy. Phys. Rev. Lett. 109, 197402 (2012). 
9. Stich, D. et al. Triplet-triplet exciton dynamics in single-walled carbon nanotubes. Nat. Photonics 8, 139-144 (2014).

10. Gabor, N. M. et al. Extremely efficient multiple electron-hole pair generation in carbon nanotube photodiodes. Science 325, 1367-1371 (2009).

11. Chen, J. et al. Bright infrared emission from electrically induced excitons in carbon nanotubes. Science 310, 1171-1174 (2005).

12. Wang, F. et al. Wideband-tuneable nanotube mode-locked fibre laser. Nat. Nanotechnol. 3, 738-742 (2008).

13. $\mathrm{Ma}, \mathrm{X}$. et al. Room-temperature single-photon generation from solitary dopants of carbon nanotubes. Nat. Nanotechnol. 10, 671-675 (2015).

14. Graf, A. et al. Electrical pumping and tuning of exciton-polaritons in carbon nanotube microcavities. Nat. Mater. 16, 911-917 (2017).

15. Avouris, P., Freitag, M. \& Perebeinos, V. Carbon-nanotube photonics and optoelectronics. Nat. Photonics 2, 341-350 (2008).

16. Sharma, A., Singh, V., Bougher, T. L. \& Cola, B. A. A carbon nanotube optical rectenna. Nat. Nanotechnol. 10, 1027-1032 (2015).

17. Pyatkov, F. et al. Cavity-enhanced light emission from electrically driven carbon nanotubes. Nat. Photonics 10, 420-427 (2016).

18. Dang, X. et al. Virus-templated self-assembled single-walled carbon nanotubes for highly efficient electron collection in photovoltaic devices. Nat. Nanotechnol. 6, 377-384 (2011).

19. Barone, P. W., Baik, S., Heller, D. A. \& Strano, M. S. Near-infrared optical sensors based on single-walled carbon nanotubes. Nat. Mater. 4, 86-92 (2005).

20. Heinz, T. F. Rayleigh scatteing spectroscopy. Carbon Nanotubes. 111, 353-369 (2007). Springer.

21. Malic, E. et al. Theory of Rayleigh scattering from metallic carbon nanotubes. Phys. Rev. B. 77, 045432 (2008).

22. Fujiwara, H. et al. Spectroscopic Ellipsometry: Principles and Applications. (John Wiley \& Sons: England, 2007).

23. Shen, Y.-R. et al. The Principles of Nonlinear Optics. (Wiley-Interscience, New York, 1984).

24. Berciaud, S. et al. Absorption spectroscopy of individual single-walled carbon nanotubes. Nano. Lett. 7, 1203-1207 (2007).

25. Blancon, J. C. et al. Direct measurement of the absolute absorption spectrum of individual semiconducting single-wall carbon nanotubes. Nat. Commun. 4, 2542 (2013).

26. Liu, K. H. et al. Systematic determination of absolute absorption cross-section of individual carbon nanotubes. PNAS 111, 7564-7569 (2014).

27. Lindfors, K., Kalkbrenner, T., Stoller, P. \& Sandoghdar, V. Detection and spectroscopy of gold nanoparticles using supercontinuum white light confocal microscopy. Phys. Rev. Lett. 93, 037401 (2004).

28. Bohren, C. F., \& Huffman, D. R. Absorption and Scattering of Light by Small Particles. (John Wiley \& Sons, New York, 2008).

29. Jan, C. M., Lee, Y. H., Wu, K. C. \& Lee, C. K. Integrating fault tolerance algorithm and circularly polarized ellipsometer for point-of-care applications. Opt. Express 19, 5431-5441 (2011).

30. Sherson, J. F. et al. Quantum teleportation between light and matter. Nature 443, 557-560 (2006).

31. Mak, K. F., McGill, K. L., Park, J. \& McEuen, P. L. The valley Hall effect in $\mathrm{MoS}_{2}$ transistors. Science 344, 1489-1492 (2014).

32. Yoshikawa, N., Tamaya, T. \& Tanaka, K. High-harmonic generation in graphene enhanced by elliptically polarized light excitation. Science 356, 736-738 (2017).

33. Lefebvre, J. \& Finnie, P. Polarized light microscopy and spectroscopy of individual single-walled carbon nanotubes. Nano. Res. 4, 788-794 (2011).

34. Liu, K. et al. High-throughput optical imaging and spectroscopy of individual carbon nanotubes in devices. Nat. Nanotechnol. 8, 917-922 (2013).

35. Islam, M. F. et al. Direct measurement of the polarized optical absorption cross section of single-wall carbon nanotubes. Phys. Rev. Lett. 93, 037404 (2004).

36. Murakami, Y., Einarsson, E., Edamura, T. \& Maruyama, S. Polarization dependence of the optical absorption of single-walled carbon nanotubes. Phys. Rev. Lett. 94, 087402 (2005).

37. Liu, K. et al. An atlas of carbon nanotube optical transitions. Nat. Nanotechnol. 7, 325-329 (2012).

38. Htoon, H., O'Connell, M. J., Doorn, S. K. \& Klimov, V. I. Single carbon nanotubes probed by photoluminescence excitation spectroscopy: the role of phonon-assisted transitions. Phys. Rev. Lett. 94, 127403 (2005).
39. Chou, S. G. et al. Phonon-assisted excitonic recombination channels observed in DNA-wrapped carbon nanotubes using photoluminescence spectroscopy. Phys. Rev. Lett. 94, 127402 (2005).

40. Perebeinos, V., Tersoff, J. \& Avouris, P. Electron-phonon interaction and transport in semiconducting carbon nanotubes. Phys. Rev. Lett. 94, 086802 (2005).

41. Miyauchi, Y. \& Maruyama, S. Identification of an excitonic phonon sideband by photoluminescence spectroscopy of single-walled carbon-13 nanotubes. Phys. Rev. B. 74, 035415 (2006).

42. Yu, N. F. et al. Light propagation with phase discontinuities: generalized laws of reflection and refraction. Science 334, 333-337 (2011).

43. Hwang, Y. \& Davis, T. J. Optical metasurfaces for subwavelength difference operations. Appl. Phys. Lett. 109, 181101 (2016).

44. Huang, L. L. et al. Three-dimensional optical holography using a plasmonic metasurface. Nat. Commun. 4, 2808 (2013).

45. Liu, K. H. et al. Van der Waals-coupled electronic states in incommensurate double-walled carbon nanotubes. Nat. Phys. 10, 737-742 (2014).

\section{Acknowledgements}

The authors thank for fruitful discussion with Wentao Yu, Dongxue Chen, and Feng Yang. This work was supported by National Key R\&D Program of China (2016YFA0300903, 2016YFA0300804), NSFC (51522201, 11474006, 51672007 and 51502007), National Equipment Program of China (ZDYZ2015-1), Beijing Graphene Innovation Program (Z161100002116028), Guangdong Innovative and Entrepreneurial Research Team Program (2016ZT06D348), Science Technology and Innovation Commission of Shenzhen Municipality (ZDSYS20170303165926217 and JCYJ20170412152620376), and the National Program for Thousand Young Talents of China.

\section{Author contributions}

K.L. and F.Y. conceived the project. K.L., F.W., E.W. and D.Y. supervised the project. F.Y. and K.L. performed optical experiments. C.L., Q.Z., S.Z., M.W. and J.Z. grew the carbon nanotubes. C.C., F.Y. and J.L. performed theoretical analysis. K.L., F.Y., F.X, J.Z., S.M. and Z.S. analyzed the experimental data. K.L., P.G. and X.B. carried out the TEM experiments. All of the authors discussed the results and wrote the paper.

\section{Additional information}

Supplementary Information accompanies this paper at https://doi.org/10.1038/s41467 018-05932-9.

Competing interests: The authors declare no competing interests.

Reprints and permission information is available online at http://npg.nature.com/ reprintsandpermissions/

Publisher's note: Springer Nature remains neutral with regard to jurisdictional claims in published maps and institutional affiliations.

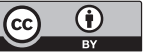

Open Access This article is licensed under a Creative Commons Attribution 4.0 International License, which permits use, sharing, adaptation, distribution and reproduction in any medium or format, as long as you give appropriate credit to the original author(s) and the source, provide a link to the Creative Commons license, and indicate if changes were made. The images or other third party material in this article are included in the article's Creative Commons license, unless indicated otherwise in a credit line to the material. If material is not included in the article's Creative Commons license and your intended use is not permitted by statutory regulation or exceeds the permitted use, you will need to obtain permission directly from the copyright holder. To view a copy of this license, visit http://creativecommons.org/ licenses/by/4.0/.

(C) The Author(s) 2018 